\title{
Chimpanzees do not take into account what others can hear in a competitive situation
}

\author{
Juliane Bräuer $\cdot$ Josep Call $\cdot$ Michael Tomasello
}

Received: 21 January 2006 / Revised: 17 April 2007 / Accepted: 17 May 2007 / Published online: 9 June 2007

(C) Springer-Verlag 2007

\begin{abstract}
Chimpanzees (Pan troglodytes) know what others can and cannot see in a competitive situation. Does this reflect a general understanding the perceptions of others? In a study by Hare et al. (2000) pairs of chimpanzees competed over two pieces of food. Subordinate individuals preferred to approach food that was behind a barrier that the dominant could not see, suggesting that chimpanzees can take the visual perspective of others. We extended this paradigm to the auditory modality to investigate whether chimpanzees are sensitive to whether a competitor can hear food rewards being hidden. Results suggested that the chimpanzees did not take what the competitor had heard into account, despite being able to locate the hiding place themselves by the noise.
\end{abstract}

Keywords Social cognition · Food competition · Perspective taking

\section{Inroduction}

Hare (2001) argued that primates may best demonstrate sophisticated cognitive abilities in test settings that are most relevant to them. As food competition is ubiquitous in the social life of chimpanzees and other primates (Sterck et al. 1997), Hare et al. (2000) devised an experimental paradigm in which two chimpanzees spontaneously competed with one another over monopolizable food. This paradigm was used to examine whether the chimpanzees could take the visual perspective of others (an ability chimpanzees do not demonstrate in other experimental paradigms such as Povi-

J. Bräuer $(\bowtie) \cdot$ J. Call $\cdot$ M. Tomasello

Max Planck Institute for Evolutionary Anthropology,

Deutscher Platz 6, 04103 Leipzig, Germany

e-mail: jbraeuer@eva.mpg.de nelli et al. 1996; Call et al. 1998; Itakura et al. 1999; Call et al. 2000; Povinelli et al. 1990). In the series of experiments by Hare and colleagues a dominant and a subordinate chimpanzee competed for two pieces of food: one piece that could be seen by both individuals, and one that could only be seen by the subordinate individual because a barrier was blocking the dominant's visual access to it. Subordinates preferred to pursue the hidden food, suggesting that they were sensitive to what others can and cannot see. A series of control experiments ruled out several of the most obvious alternative explanations. Although Karin-D'Arcy and Povinelli (2002) could not replicate this finding, the chimpanzees in that study competed in a much smaller space than those in the original study. Bräuer et al. (2007) argued that it is important to have an appropriate level of competition between the chimpanzees to encourage the use of their perspective taking abilities, and suggested that spatial arrangement of the competition arena is a major factor influencing competition intensity. By using a test setup that encouraged competition, Bräuer and colleagues found results consistent with Hare et al. (2000) in a new group of chimpanzees: subordinates preferentially targeted the hidden pieces that the dominants could not see.

In the current study we investigate whether this basic social-cognitive skill would generalize beyond the visual modality to other kinds of perspective taking. Specifically, do chimpanzees take into account what a competitor has heard? There is some evidence that wild chimpanzees remain silent when patrolling their borders (Boesch et al. 2000), and female chimpanzees suppress copulation calls when mating with subordinate animals in the vicinity of dominant males (Hauser 1990). Experimental results have further reinforced the idea that chimpanzees are sensitive to what others can hear-or at least to the behavioral consequences of making sounds. In a study by Melis et al. (2006), 
a human experimenter competed with a chimpanzee over two pieces of food. The experimenter could retract the food if she heard the subject trying to take it. The chimpanzee could open one of two doors to get access to one of the food pieces (one piece behind each door), but one door made a loud noise when it was opened. It is important to emphasize that it was the chimpanzees' actions that created the sound. Subjects preferentially reached through the silent tunnelsuccessfully concealing their taking of the food from the human competitor. A study with rhesus monkeys (Santos et al. 2006) found similar results.

In the current study we used the same chimpanzees as in the Bräuer et al. (2007) study. The methods were also identical to experiment 2 of that study except in two respects: the food was always hidden for the dominant competitor, and we varied whether the food was baited with or without a noise. Following the previous findings, we expected that subordinate chimpanzees would not approach hidden food that was baited with noise, as the competitor could have also heard the noise and thus would be likely to approach the location herself. However, an important difference between this and previous studies was that the noise was made by the experimenter, and not by the subject. Thus, if chimpanzees could avoid the noisy piece of food, it would suggest that their perspective-taking skills extend beyond the visual domain.

\section{Methods}

Eleven adult and subadult chimpanzees (6-27 years old) participated in this study. All subjects lived in a single group consisting of 17 conspecifics in the Wolfgang Köhler Primate Center in the Leipzig Zoo (Germany).

Testing took place in three adjacent cages: the cage for the dominant chimpanzee on the left, the cage for the subordinate subject on the right, and the testing cage $(2.5 \mathrm{~m} \times 2.6 \mathrm{~m} \times 2.5 \mathrm{~m})$ in the middle. The mesh between the cages was blocked by black Plexiglas panels, making it impossible to see from one cage into the others. The guillotine doors into the middle cage were in the middle of the left and right cages. We used two upside-down buckets $(23 \mathrm{~cm}$ high, diameter of $28 \mathrm{~cm}$ ) as barriers. They were placed on the extreme sides of the cage, $2 \mathrm{~m}$ away from the subordinates' door and $2 \mathrm{~m}$ apart from each other. On the top of each bucket was a plastic plate (diameter of $30 \mathrm{~cm}$ ), and another plate of the same size was half under each bucket, so that it would be possible to place the food noisily behind the bucket (from the dominant's view, on the plate on the floor). Pieces of banana were used for the food competition.

Each subject was paired with a dominant individual, with dominance determined by three trials of a food competition test over banana halves before the test sessions began.
At the beginning of each test session the dominant individual was in the left cage and the subordinate was in the right cage; both doors were raised slightly so that the two opponents could see each other. After closing the doors the experimenter entered the testing cage to put the buckets and the food in place according to condition. A test trial started when the door of the subordinate was opened a bit so that she could see the buckets and the food. Then the door was closed again and immediately opened so that the subordinate could enter the cage. The door of the dominant animal was opened when the subordinate subject had entered the cage. Thus, the subordinates could not simply react to dominants' behavior, but the head start was not long enough to grab the food before dominant entered.

In the three experimental conditions, subjects always could see the food. The dominant, in contrast, could never see the food, but sometimes could hear how it was baited. The conditions were determined by where and how (with or without noise) the food was placed:

Hidden-Hidden (noise) Two pieces were placed behind the two buckets. One piece was placed in a very noisy manner, as E rumpled a plastic bag and put the food on the plate with a bang. The dominant had heard only the placing of one of the two food pieces.

Hidden1 (noise) There was only one piece that was placed very noisily behind one bucket. The dominant had heard the placing of the food.

Hiddenl There was only one piece that was placed behind one bucket. The dominant could not see or hear the placement of the food.

Competitive pairs were created by pairing a dominant with a subordinate individual. The subject of the experiment was the subordinate. Each pair of animals was tested in two trials in each condition, with order of trials randomized across subjects. A daily session usually consisted of the six experimental trials (and six related trials that are not discussed in this paper) for a given pair. A subject was only paired with one dominant chimpanzee per day, so it never experienced more than 12 trials per day. If either the dominant or the subordinate individual did not get any food for three trials in a row, we interspersed a filler-trial in which that animal got both pieces. This was to increase motivation to approach the food, as well as to reduce any potential biases on the part of the dominant (e.g., always shadowing the subordinate). Pairs were only included in the analysis if the dominance relationship was clear, and the subordinate individual still sometimes went for the food during a given session (for the numbers of pairs for each subject, see Table 1). Thus we analyzed 27 pairs with 8 animals playing the role of the subordinate subject. 
Table 1 Percentages of trials that subjects reached for (experimental conditions) and approached (control condition) a Ordered from high ranking to low ranking

\begin{tabular}{|c|c|c|c|c|c|}
\hline \multirow{2}{*}{$\begin{array}{l}\text { Subject }^{\text {a }} \\
\text { (tested in } N \text { pairs) }\end{array}$} & \multicolumn{2}{|c|}{ Hidden-Hidden (noise) } & \multirow{2}{*}{$\begin{array}{l}\text { Hidden } 1 \\
\text { Reach for } \\
\text { hidden piece }\end{array}$} & \multirow{2}{*}{$\begin{array}{l}\text { Hidden1 (noise) } \\
\text { Reach for } \\
\text { noisy piece }\end{array}$} & \multirow{2}{*}{$\begin{array}{l}\text { Control } \\
\text { Approach } \\
\text { noisy piece }\end{array}$} \\
\hline & $\begin{array}{l}\text { Reach for } \\
\text { hidden piece }\end{array}$ & $\begin{array}{l}\text { Reach for } \\
\text { noisy piece }\end{array}$ & & & \\
\hline Fraukje (1) & 0 & 0.5 & 0.5 & 0.5 & 0.33 \\
\hline Ulla (3) & 0.5 & 0 & 0.5 & 0.66 & 0.5 \\
\hline Frodo (1) & 0.5 & 0 & 0 & 0.5 & 1.0 \\
\hline Jahaga (2) & 0 & 0.25 & 0.5 & 0.5 & 0.83 \\
\hline Fifi (4) & 0.5 & 0.38 & 0.75 & 0.63 & 0.83 \\
\hline Sandra (6) & 0.25 & 0.33 & 0.33 & 0.66 & 0.5 \\
\hline Gertruida (5) & 0.2 & 0.1 & 0.5 & 0.2 & 0.66 \\
\hline Patrick (5) & 0.4 & 0.2 & 0.3 & 0.2 & 0.83 \\
\hline All & 0.29 & 0.22 & 0.42 & 0.48 & 0.68 \\
\hline
\end{tabular}

After we tested the pairs we presented all chimpanzees that were involved with a control condition. They were tested for six consecutive trials in a row using a similar setup as in the previous conditions:

Control: only the subject was present. One piece was placed very noisily behind one of the two buckets. Thus, subject could not see the food but she could hear how it was baited.

This control was designed to ensure that these chimpanzees were able localize the noise, and would go for the noisy piece when they could not see the food.

All trials were scored from the videotapes. For each experimental trial we scored whether the subject obtained the food and whether the subject reached for the food (i.e., raised an arm in the direction of the food, before the dominant had approached any bucket). For each subject we calculated the percentage of reaching for each type of food (noisy or silent) as the average for that subject across all pairs in which she played the subordinate role. For the control condition we scored whether the animal would approach the piece of food. An independent observer scored a randomly selected sample of $20 \%$ of the trials to assess inter-observer reliability for reaching in the experimental trials (Cohen's Kappa $=0.86, N=14$ ) and approaching in the control trials (Cohen's Kappa $=1.00, N=33$ ).

\section{Results}

Subjects in the Hidden-Hidden (noise) condition did not reach significantly more often for the silent piece compared to the noisy piece (Wilcoxon $T=23, N=8, P=0.539$ ). The comparison between the conditions Hidden 1 (noise) and Hidden 1 revealed similar results: subjects did not reach more often for the silent pieces than for the noisy ones (Wilcoxon $T=14, N=6, P=0.563$ ). In contrast, animals were able to localize the noise for themselves as they significantly approached the correct bucket-the one with the noisy food-in the control condition (Wilcoxon against chance $T=34, N=8, P=0.031$ see Table 1$)$.

Overall subordinates obtained $39 \%$ of the food in the Hidden 1, 35\% in the Hidden 1 (noise) and 45\% HiddenHidden (noise) condition.

\section{Discussion}

We found no evidence that subordinate chimpanzees took into account whether or not the dominant heard the food being hidden. Subjects did not avoid the noisy piece in either the within trial or the between-trial comparisons. However, they could use the noise to localize the food and acquire it for themselves. Moreover, we can rule out that the level of competition between the chimpanzees was not appropriate to foster the use of their cognitive skills, as these subjects did show visual perspective taking abilities in the same setup (Bräuer et al. 2007).

Why did subordinates not avoid the piece of food that had been placed noisily such that the dominant could know where it was? Previously, Call (2004) found that chimpanzees are able localize food by using noise, much like the chimpanzees tested here. In his experiment, he found that chimpanzees can infer in which of two cups that food is located after hearing both cups being shaken. However, chimpanzees do not normally experience food that is noisy (though this is not necessarily true of wild chimpanzees who hunt noisy colobus monkeys, Boesch et al. 2000). Consequently, this test may simply be an unusual situation for them: although they expect that visual information is relevant in competitive contexts, they do not spontaneously take noise into account when competing with a conspecific.

Another interpretation of the current findings is that chimpanzees' knowledge about the perception of others is 
limited to the visual domain. They simply may not understand that a competitor uses its auditory perception to localize things. However, Melis et al. (2006) showed that chimpanzees are sensitive to both what others can see and what others can hear-indeed, chimpanzees in her experiment were able to engage in concealment, a simple form of deception, based on their understanding of others' auditory perceptions. There are three important differences between that study and the current study that may account for these conflicting results. First, in the Melis et al. experiment, subjects produced the noise when they selected the noisy tunnel; in the current study a third party produced the noise. This is analogous to the examples observed in the wild in which individuals inhibit calls while patrolling their borders (Boesch et al. 2000) or copulating (Hauser 1990). Being the noise producer, as opposed to noise receptor, may simplify the problem, as the former entails a dyadic relation in which the subject plays an active role, while the latter reflects a triadic relation in which the subject merely plays a passive role. Moreover, if the noise is one that individuals themselves produce, chimpanzees may have just learnt the behavioral consequences of making sounds in certain situations, rather than having a full understanding of what others can hear.

Second, in our study the noise occurred during the baiting of the buckets. This means that subordinate subjects not only had to localize the noise itself, but also keep in mind what the dominant had heard in the recent past. Although chimpanzees show sensitivity for what conspecifics have seen (Hare et al. 2001), this makes the task much more difficult and could be a reason why they did not avoid the noisy piece. Finally, in the study by Melis et al. (2006), subjects competed with a human rather than a conspecific, and they could have learned through their daily interactions that humans, unlike conspecifics, attend to and care about noise in competitive situations.

At the moment, we cannot distinguish which of these alternatives accounts for the contrasting results. We found no evidence that chimpanzees take into account what others can hear in a competitive situation when they did not produce the noise. Future studies should address whether they are sensitive to what others can hear, or whether they just learn to avoid making a noise in certain situations. This could be tested by varying whether the chimpanzee controls the noise production, as well as whether the chimpanzee competes with a human or a conspecific.

Acknowledgments We thank Julia Riedel, Hagen Lehmann and Katrin Schumann for helping with data collection, and Tanja Lindner, Josefine Kalbitz and Kathrin Greve for coding.

\section{References}

Boesch C, Boesch-Achermann H (2000) The chimpanzees of the Tai Forest. University Press, Oxford

Bräuer J, Call J, Tomasello M (2007) Chimpanzees really know what others can see in a competitive situation. Anim Cogn: doi $10.1007 / \mathrm{s} 10071-007-0088$

Call J (2004) Inferences about the location of food in the great apes. J Comp Psychol 118:232-241

Call J, Hare BA, Tomasello M (1998) Chimpanzee gaze in an objectchoice task. Anim Cogn 1:89-99

Call J, Agnetta B, Tomasello M (2000) Cues the chimpanzees do and do not use to find hidden objects. Anim Cogn 3:23-34

Hare B (2001) Can competitive paradigms increase the validity of experiments on primate social cognition? Anim Cogn 4:269280

Hare B, Call J, Agnetta B, Tomasello M (2000) Chimpanzees know what conspecifics do and do not see. Anim Behav 59:771-785

Hare B, Call J, Tomasello M (2001) Do chimpanzees know what conspecifics know? Anim Behav 61:139-151

Hauser MD (1990) Do chimpanzee copulatory calls incite male-male competition? Anim Behav 39:596-597

Itakura S, Agnetta B, Hare B, Tomasello M (1999) Chimpanzee use of human and conspecific social cues to locate hidden food. Dev Sci 2:448-456

Karin-D'Arcy M, Povinelli DJ (2002) Do chimpanzees know what each other see? A closer look. Int J Comp Psychol 15:21-54

Melis AP, Call J, Tomasello M (2006) Chimpanzees (Pan troglodytes) conceal visual and auditory information from others. J Comp Psychol 120:154-162

Povinelli DJ, Eddy TJ (1996) Factors influencing young chimpanzees' (Pan troglodytes) recognition of attention. J Comp Psychol 110:336-345

Povinelli DJ, Nelson KE, Boysen ST (1990) Inferences about guessing and knowing by chimpanzees (Pan troglodytes). J Comp Psychol 104:203-210

Santos LR, Nissen AG, Ferrugia JA (2006) Rhesus monkeys, Macaca mulatta, know what others can and cannot hear. Anim Behav 71:1175-1181

Sterck EHM, Watts DP, van Schaik CP (1997) The evolution of female social relationships in nonhuman primates. Behav Ecol Sociobiol 41:291-309 